\section{Deep sequencing of bone marrow microenvironments of patients with del $(5 q)$ myelodysplastic syndrome reveals imprints of antigenic selection as well as generation of novel T-cell clusters as a response pattern to lenalidomide}

Thorben Mährle, ${ }^{1 \star}$ Nuray Akyüz, ${ }^{1 *}$ Pim Fuchs, ${ }^{2}$ Nicola Bonzanni, ${ }^{2}$ Donjete Simnica, ${ }^{1,3}$ Ulrich Germing, ${ }^{4}$ Anne Marie Asemissen, ${ }^{1}$ Johann Christoph Jann, ${ }^{5}$ Florian Nolte, ${ }^{5}$ Wolf-Karsten Hofmann, ${ }^{5}$ Daniel Nowak ${ }^{5}$ and Mascha Binder ${ }^{1,3}$

${ }^{1}$ Department of Oncology and Hematology, BMT with Pneumology section, Hubertus Wald Tumorzentrum / UCCH, University Medical Center Hamburg-Eppendorf, Hamburg, Germany; ' ${ }^{2}$ NPICOM, 's-Hertogenbosch, the Netherlands; ${ }^{3}$ Department of Hematology and Oncology, University Hospital Halle (Saale), Germany; ${ }^{4}$ Department of Hematology, Oncology, and Clinical Immunology, Heinrich Heine University Düsseldorf, Germany and ${ }^{5}$ Department of Hematology and Oncology, Medical Faculty Mannheim of the Heidelberg University, Mannheim, Germany

*TM and NA contributed equally to this work.

\section{ABSTRACT}

$\tau$ n myelodysplastic syndromes with a partial deletion of the long arm of chromosome 5 , del $(5 q)$, lenalidomide is believed to reverse anergic T-cell immunity in the bone marrow resulting in suppression of the del(5q) clone. In this study we used next-generation sequencing of immunoglobulin heavy chain $(I G H)$ and T-cell receptor beta $(T R B)$ rearrangements in bone marrow-residing and peripheral blood-circulating lymphocytes of patients with del(5q) myelodysplastic syndromes to assess the immune architecture and track adaptive immune responses during treatment with lenalidomide. The baseline bone marrow B-cell space in patients was comparable to that of age-matched healthy controls in terms of gene usage and IGH clonality, but showed a higher percentage of hypermutated IGH sequences, indicating an expanded number of antigen-experienced B lineage cells. Bone marrow B lineage clonality decreased significantly and hypermutated IGH clones normalized upon lenalidomide treatment, well in line with the proliferative effect on healthy antigen-inexperienced B-cell precursors previously described for this drug. The T-cell space in bone marrow of patients with del(5q) myelodysplastic syndromes showed higher TRB clonality compared to that of healthy controls. Upon lenalidomide treatment, myelodysplastic syndrome-specific T-cell clusters with low to medium spontaneous generation probabilities emerged; these clusters were shared across patients, indicating a common antigen-driven $\mathrm{T}$-cell response pattern. Hence, we observed $B$ lineage diversification and generation of new, antigen-dependent T-cell clusters, compatible with a model of adaptive immunity induced against the del $(5 \mathrm{q})$ clone by lenalidomide. Overall, this supports the concept that lenalidomide not only alters the functional T-cell state, but also the composition of the $\mathrm{T}$ - and $\mathrm{B}$-cell repertoires in $\operatorname{del}(5 \mathrm{q})$ myelodysplastic syndromes.

\section{Introduction}

Myelodysplastic syndromes (MDS) are clonal stem cell disorders characterized by inefficient hematopoiesis. The most prevalent cytogenetic abnormality in these diseases is a partial deletion of the long arm of chromosome 5, del(5q), which is present in about $15 \%$ of patients. ${ }^{1}$ This clinically distinct entity is char-
Haematologica 2019

Volume 104(7):1355-1364

\section{Correspondence:}

MASCHA BINDER,

mascha.binder@uk-halle.de

Received: October 8, 2018.

Accepted: January 15, 2019.

Pre-published: January 24, 2019.

doi:10.3324/haematol.2018.208223

Check the online version for the most updated information on this article, online supplements, and information on authorship \& disclosures: www.haematologica.org/content/104/7/1355

(C)2019 Ferrata Storti Foundation

Material published in Haematologica is covered by copyright. All rights are reserved to the Ferrata Storti Foundation. Use of published material is allowed under the following terms and conditions:

https://creativecommons.org/licenses/by-nc/4.0/legalcode. Copies of published material are allowed for personal or internal use. Sharing published material for non-commercial purposes is subject to the following conditions: https://creativecommons. org/licenses/by-nc/4.0/legalcode sect. 3. Reproducing and sharing published material for commercial purposes is not allowed without permission in writing from the publisher. 
acterized by refractory anemia, often thrombocythemia with atypical megakaryocytes in the bone marrow (BM) and a relatively low progression rate to acute myeloid leukemia compared to the rate of transformation of other types of $\mathrm{MDS}^{2}$ The pathogenesis seems to be partially driven by haploinsufficiency of genes located in the deleted area on chromosome $5^{3-8}$ Moreover, there is evidence of adaptive and innate immune dysregulation with altered cytokine networks, diminished NK cell activity and an anergic $\mathrm{T}$-cell environment failing to suppress the defective del $(5 q)$ clone. $^{9}$

In 2005, lenalidomide was approved in the USA for the treatment of lower-risk, transfusion-dependent $\operatorname{del}(5 \mathrm{q})$ MDS patients as the first immunomodulatory drug. In the placebo-controlled phase III trial MDS-004, 56\% of patients who received $10 \mathrm{mg} /$ day lenalidomide achieved red blood cell transfusion independency for more than 26 weeks. ${ }^{10}$ Response to lenalidomide was shown to be associated with significant improvement in survival with a median overall survival of 4 years. ${ }^{11}$ Cereblon (CRBN), a substrate receptor of the CRL4 ubiquitin ligase complex, is the primary molecular target through which lenalidomide mediates its anti-tumor effects. Krönke et al. demonstrated that lenalidomide induces the ubiquitination and degradation of IKZF1, IKZF3 and CK1 $\alpha$ by binding to CRBN..$^{12,13}$ $\mathrm{CK} 1 \alpha$, a negative regulator of $\mathrm{p} 53$, may be of particular pathophysiological relevance in the context of MDS $\operatorname{del}(5 \mathrm{q})$, since it is encoded by a gene within the deleted chromosomal region and haploinsufficient expression sensitizes cells to lenalidomide therapy. ${ }^{12}$ Furthermore, the ubiquitin-independent physiological chaperone-like function of CRBN is targeted by lenalidomide. Eichner et al. found that lenalidomide outcompetes CRBN for binding to and stabilizing the CD147-MCT1 complex. ${ }^{14}$ This complex promotes various biological functions, including angiogenesis, proliferation and invasion. Since recent evidence suggests a negative regulatory role of CD147 in Tcell-mediated immune responses, ${ }^{15-17}$ lenalidomideinduced CD147 destabilization has been speculated to explain not only direct anti-tumor effects, but also immune cell activation. ${ }^{14}$ Functionally, the immunomodulation induced by lenalidomide was described as a reversal of pathological T-cell tolerance by increasing T-cell effector function and secretion of interleukin-2, interferon- $\gamma$ and tumor necrosis factor- $\alpha$ by $\mathrm{CD}^{+}$and $\mathrm{CD}^{+} \mathrm{T}$ cells. ${ }^{18}$ Expanding on this, a recent RNA sequencing study on non-del(5q) MDS patients suggested that the immunomodulatory signal delivered by lenalidomide may also affect the composition rather than only the functional state of the local BM immune cells. ${ }^{19}$ However, the immunomodulatory effects of lenalidomide on the microenvironment have not yet been fully defined.

In this study, we analyzed local BM and peripheral blood (PB) environments to understand the clonal immune architecture of $\mathrm{MDS} \operatorname{del}(5 \mathrm{q})$ and its modulation by lenalidomide. To do this, we used state-of-the-art next-generation sequencing (NGS) of immunoglobulin heavy chain $(I G H)$ and T-cell receptor beta (TRB) rearrangements as an advanced technology that enables simultaneous identification of tens of thousands of unique $\mathrm{B}$-cell and $\mathrm{T}$-cell receptor rearrangements from a single tissue sample as well as tracking over time. These analyses demonstrated that lenalidomide shapes T-cell adaptive immune responses in the $\mathrm{BM}$ niche of patients with $\operatorname{MDS} \operatorname{del}(5 q)$.

\section{Methods}

\section{Patients' characteristics}

Patients' BM samples were collected between 2013 and 2016 at baseline and after a median of 12 months of lenalidomide treatment in the course of the LE-MON 5 and Bioregister trials. The cohort comprised 15 patients with $\operatorname{del}(5 q)$ MDS. Matching preand post-treatment $\mathrm{PB}$ samples were available for eight of these patients. All del(5q) patients were treated in the LE-MON 5 trial, ${ }^{20}$ a German multicenter, single-arm, open-label, phase II study [Safety of Lenalidomide Monotherapy and Markers for Disease Progression in Patients With IPSS Low- or Intermediate-1 Risk Myelodysplastic Syndromes (MDS) Associated With an Isolated Deletion 5q Cytogenetic Abnormality] according to the standard lenalidomide protocol. ${ }^{10}$ As a reference, seven BM and 22 PB samples from age-matched healthy controls without hematologic abnormalities were included. Five BM samples were collected during hip replacement surgery and two were obtained from iliac crest aspiration of healthy individuals, without apparent differences. Hemograms of all the healthy donors were normal as were their C-reactive protein levels. The BM of all MDS patients was obtained by standardized aspiration and biopsy of the iliac crest. All MDS del $(5 q)$ samples analyzed in this study were previously subjected to NGS of TP53, ${ }^{21}$ and were negative for TP53 mutations. The patients' characteristics are shown in Table 1.

\section{Study approval}

Informed consent was obtained from all patients and healthy controls for the use of their diagnostic material for scientific purposes approved by the institutional review board (Ethikkommission der Medizinischen Fakultät, Henrich Heine University Düsseldorf, Germany, project number MC-LKP-392).

\section{Isolation of genomic DNA}

Genomic DNA was isolated from $>10^{6}$ frozen $\mathrm{BM}$ or $\mathrm{PB}$ mononuclear cells using a Gen Elute mammalian genomic DNA miniprep kit (Sigma-Aldrich, Taufkirchen, Germany) according to the supplier's suggestions.

\section{Multiplex polymerase chain reaction analysis for IGH and TRB repertoire amplification for Illumina targeted next-generation sequencing}

Both the IGH and TRB genes containing the entire rearranged $V, D$ and $J$ segments were amplified with BIOMED2-FR1 (IGH), -TRB-A and -B primer pools starting from $250 \mathrm{ng}$ of input genomic DNA.22 Amplicons were tagged with Illumina adapters and indices in two consecutive polymerase chain reactions (PCR), as previously described. ${ }^{23}$ Briefly, previously published primers $^{22-24}$ for the first PCR annealed within either the $I G H$ or $T R B$ loci and contained Illumina-compatible adapters for NGS primer annealing. A second PCR was performed using outer primers to extend the Illumina adapter for later hybridization of amplicons to the Illumina flow cell and to add a seven nucleotide barcode, allowing each sequence to be matched during data analysis to a certain patient and time point. All primers were purchased from Metabion (Martinsried, Germany). All PCR were performed using Phusion HS II polymerase (Thermo Fisher Scientific Inc., Darmstadt, Germany). Amplicons with the expected size were purified after agarose gel electrophoresis using a NucleoSpin ${ }^{\oplus}$ Gel and PCR Clean-up kit (MachereyNagel, Düren, Germany). The concentration of the amplicons was determined on Qubit (OIAGEN, Hilden, Germany) and the quality of the amplicon pools was controlled on an Agilent 2100 Bioanalyzer (Agilent technologies, Böblingen, Germany) before being subjected to NGS. 
Illumina next-generation sequencing and data analysis

NGS and demultiplexing were performed on an Illumina MiSeq sequencer (600-cycle single indexed, paired-end run, V3chemistry). Analysis of rearranged IGH and TRB loci was computed using the MiXCR and ENPICOM's ImmunoGenomix Platforms analysis tools. ${ }^{23,25}$ In silico grouping of lymphocyte interactions by paratope hotspots (GLIPH) cluster and generation probability analyses were performed as described in previous publications. ${ }^{26,27}$

\section{Repertoire metrics analyses}

Clonality is an index calculated according to the formula " 1 Pielou's evenness". ${ }^{28}$ Pielou's evenness is calculated according to the formula $\mathrm{J}=\mathrm{H}^{\prime} / \ln (\mathrm{S})$ where $\mathrm{H}^{\prime}$ is the Shannon-Wiener diversity index and $S$ is the total number of clones in a specific sample. ${ }^{29}$ The clonality index ranges from 0 to 1 , with 0 indicating complete diversity and 1 indicating absolute clonality of the investigated sample and thus the presence of only one clone.

\section{In silico GLIPH and generation probability analyses}

We applied the GLIPH algorithm to our NGS-generated healthy donor and MDS patient TRB dataset. ${ }^{26}$ Next we investi- gated the generation probability of the GLIPH-generated TRB clusters of our healthy controls and MDS patients with the IGoR algorith $\mathrm{m}^{27}$ and used the negative logarithmic value to display low probability values better. Clusters with low generation probability are expected to be generated by antigen selection, although no cutoff value defining "low" exists, to the best of our knowledge. For a better description of our data we decided to define cutoffs in our study by dividing the range between the lowest (13.1400259) and highest (36.7368006) observed generation probability values into three equally sized areas representing high (a value in the range of 13.1400259-21.0056175), medium (21.0056176-28.871209) and low (28.871210-36.7368006) probability. T-cell clusters that expand after lenalidomide treatment and have a low generation probability are expected to have been generated by antigen selection, whereas clusters which expand but have a high generation probability are less likely to have expanded by antigen selection.

\section{Statistics}

Differences in distributions were studied using the Student $t$ test. All statistical analyses were performed with GraphPad Prism 7.0 (GraphPad Software, La Jolla, CA, USA).

Table 1. Characteristics of the cohort of patients with del(5q) myelodysplastic syndromes.

\begin{tabular}{|c|c|c|c|c|c|c|c|c|}
\hline $\begin{array}{l}\text { Patient } \\
\text { n. }\end{array}$ & $\begin{array}{l}\text { Age at first } \\
\text { dilagnosis }\end{array}$ & Sex & WHO diagnosis & IPSS & Samples & $\begin{array}{l}\text { Months after } \\
\mathbf{1}^{\text {st }} \text { sample }\end{array}$ & $\begin{array}{c}\text { Response at } \\
\text { sample collection }\end{array}$ & $\begin{array}{c}\text { Best } \\
\text { response }\end{array}$ \\
\hline 1 & 66 & $\mathrm{~F}$ & Isolated del5q & Int-1 & $\mathrm{BM}, \mathrm{PB}$ & $\begin{array}{c}0 \\
12\end{array}$ & $\begin{array}{l}\text { Baseline } \\
\text { PR }\end{array}$ & $\mathrm{PR} / \mathrm{TI}$ \\
\hline 2 & 75 & $\mathrm{~F}$ & Isolated del5q & Int-1 & $\mathrm{BM}, \mathrm{PB}$ & $\begin{array}{c}0 \\
16\end{array}$ & $\begin{array}{c}\text { Baseline } \\
\text { No treatment }\end{array}$ & $\begin{array}{c}\text { No } \\
\text { change/SD }\end{array}$ \\
\hline 3 & 78 & $\mathrm{~F}$ & Isolated del5q & Int-1 & $\mathrm{BM}, \mathrm{PB}$ & $\begin{array}{l}0 \\
7\end{array}$ & $\begin{array}{l}\text { Baseline } \\
\text { PR }\end{array}$ & $\mathrm{TI}$ \\
\hline 4 & 68 & $\mathrm{~F}$ & Isolated del5q & Int-1 & BM & $\begin{array}{c}0 \\
15\end{array}$ & $\begin{array}{c}\text { Baseline } \\
\text { PD (morphology) }\end{array}$ & TI \\
\hline 5 & 74 & M & Isolated del5q & Int-1 & $\mathrm{BM}, \mathrm{PB}$ & $\begin{array}{c}0 \\
30\end{array}$ & $\begin{array}{l}\text { Baseline } \\
\text { NA }\end{array}$ & CCR/TI \\
\hline 6 & 52 & M & Isolated del5q & Int-1 & $\mathrm{BM}, \mathrm{PB}$ & $\begin{array}{l}0 \\
7\end{array}$ & $\begin{array}{l}\text { Baseline } \\
\text { SD }\end{array}$ & NA \\
\hline 7 & 71 & M & Isolated del5q & Low & $\mathrm{BM}, \mathrm{PB}$ & $\begin{array}{c}0 \\
18\end{array}$ & $\begin{array}{l}\text { Baseline } \\
\text { CR }\end{array}$ & CCR/TI \\
\hline 8 & 63 & $\mathrm{~F}$ & Isolated del5q & Int-1 & $\mathrm{BM}, \mathrm{PB}$ & $\begin{array}{l}0 \\
6\end{array}$ & $\begin{array}{l}\text { Baseline } \\
\text { SD }\end{array}$ & NA \\
\hline 9 & 76 & $\mathrm{~F}$ & Isolated del5q & Low & $\mathrm{BM}, \mathrm{PB}$ & $\begin{array}{c}0 \\
42\end{array}$ & $\begin{array}{l}\text { Baseline } \\
\text { PR/CCR? }\end{array}$ & CCR/TI \\
\hline 10 & 48 & $\mathrm{~F}$ & Isolated del5q & Int-1 & BM & $\begin{array}{c}0 \\
30\end{array}$ & $\begin{array}{l}\text { Baseline } \\
\text { Relapse }\end{array}$ & CCR/TI \\
\hline 11 & 68 & $\mathrm{~F}$ & Isolated del5q & Int-1 & BM & $\begin{array}{c}0 \\
12\end{array}$ & $\begin{array}{c}\text { Baseline } \\
\text { PD (RAEB-2) }\end{array}$ & PR/TI \\
\hline 12 & 74 & M & Isolated del5q & Int-1 & BM & $\begin{array}{l}0 \\
6\end{array}$ & $\begin{array}{c}\text { Baseline } \\
\text { PD (RAEB-2) }\end{array}$ & $\mathrm{TI}$ \\
\hline 13 & 60 & M & Isolated del5q & Int-1 & $\mathrm{BM}$ & $\begin{array}{l}0 \\
6\end{array}$ & $\begin{array}{l}\text { Baseline } \\
\text { CR }\end{array}$ & CCR/TI \\
\hline 14 & 51 & $\mathrm{~F}$ & Isolated del5q & Int-1 & BM & $\begin{array}{c}0 \\
12\end{array}$ & $\begin{array}{l}\text { Baseline } \\
\text { CR }\end{array}$ & CCR/TI \\
\hline 15 & 45 & $\mathrm{~F}$ & Isolated del5q & Int-1 & $\mathrm{BM}$ & $\begin{array}{l}0 \\
6\end{array}$ & $\begin{array}{l}\text { Baseline } \\
\text { CR }\end{array}$ & $\mathrm{CCR} / \mathrm{TI}$ \\
\hline
\end{tabular}

N: number; F: female; M: male; WHO: World Health Organization; Isolated del5q: myelodysplastic syndrome with isolated deletion of chromosome 5q; IPSS: International Prognostic Scoring System; Int-1: intermediate risk 1; BM; bone marrow; PB: peripheral blood;TI: transfusion independence; CR: complete remission; CCR: complete cytogenetic remission; PR: partial remission; SD: stable disease; PD: progressive disease; RAEB.-2: refractory anemia with excess blasts-2; NA: not available; Baseline: indicates sample taken before the initiation of lenalidomide treatment. 
A

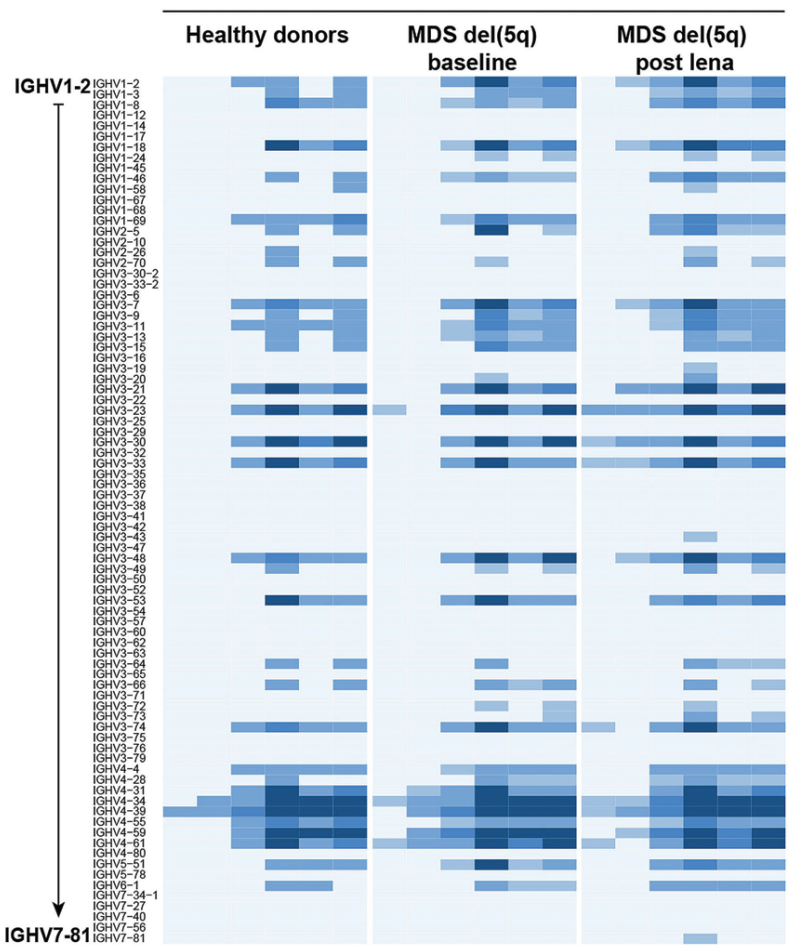

IGHJ1

C

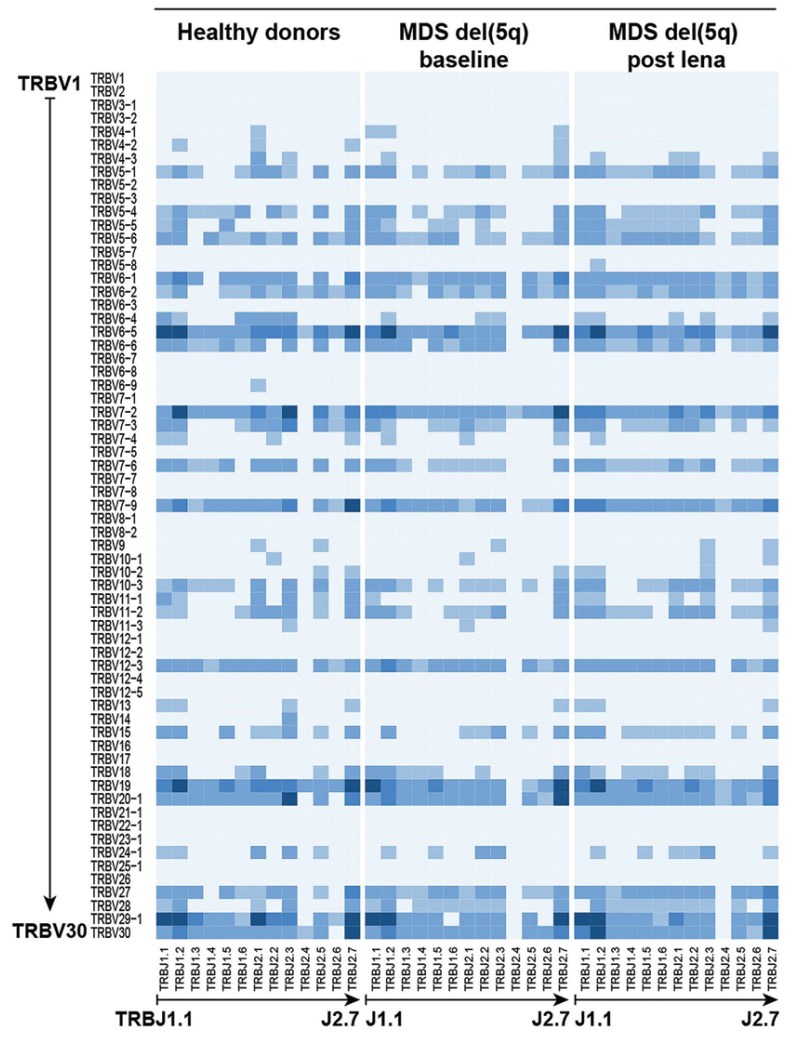

B
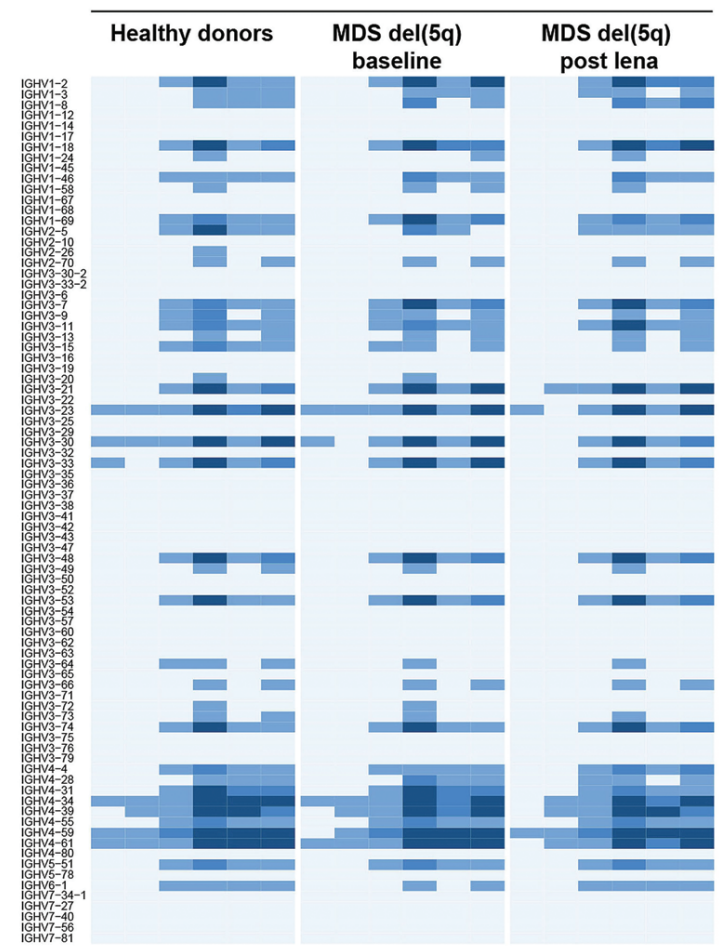

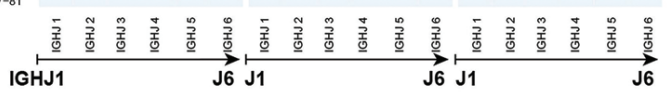

D

PB

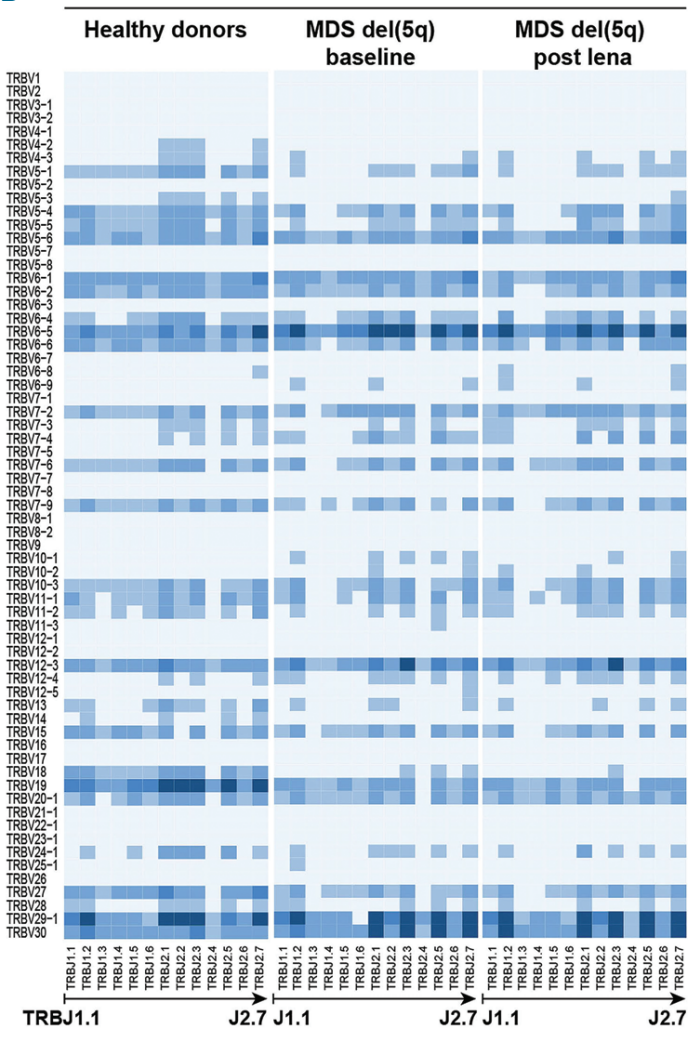

Figure 1. TRB and IGH V-and J-gene usage before and after lenalidomide treatment in patients with del(5q) myelodysplastic syndromes and in healthy controls. For each sample from healthy controls and patients with del(5q) myelodysplastic syndromes before (baseline) and after lenalidomide therapy, the frequency of the $V-J$ gene combinations of all IGH and TRB clones was calculated separately as a percentage. The median frequencies, in percentages, are shown for (A,B) IGH and (C,D) TRB V-J-gene combinations for healthy controls and patients, before and after lenalidomide treatment, in $(A, C)$ bone marrow and (B,D) peripheral blood samples as heat maps. Light blue represents lowest frequency and dark blue highest frequency. For both IGH and TRB, $J$ genes are listed along the $\mathrm{x}$ axis and functional $V$ genes along the $y$ axis. BM: bone marrow; PB: peripheral blood; MDS; myelodysplastic syndrome; baseline: prior to lenalidomide treatment; post lena: after lenalidomide treatment 


\section{Results}

No disease-specific gene usage or shared individual B- or T-cell clones in immune environments of patients with del(5q) myelodysplastic syndromes

In search of a repertoire signature of immune environments in del $(5 q) \mathrm{MDS}$ or a signature of treatment response to lenalidomide, we globally compared B- and T-cell spaces of our cohort of $\operatorname{del}(5 \mathrm{q}) \mathrm{MDS}$ patients (characteristics shown in Table 1) with those of healthy, age-matched controls. To this end, next-generation immunosequencing was performed on the T lineage TRB locus and the B lineage $I G H$ locus in all subjects at baseline as well as 6-42 months after initiation of lenalidomide treatment. As regards $T R B$ and $I G H$ gene usage, $\operatorname{del}(5 \mathrm{q})$ MDS samples did not differ from samples from age-matched healthy donors and no systematic biases in gene usage were observed upon lenalidomide treatment (Figure 1A-D). Moreover, no disease-specific shared B- or T-cell clones were discernable (Figure 2). Interestingly, $\mathrm{PB}$ and BM samples as well as follow-up samples from the same patient showed significant overlapping for TRB (Figure 2B), but not for IGH (Figure 2A).

\section{The clonal bone marrow B-cell space diversifies upon lenalidomide treatment in del $(5 q)$ myelodysplastic syndromes}

Next, we explored B-cell architectural changes upon lenalidomide treatment by comparing pre- and postlenalidomide $I G H$ repertoires. The pre-lenalidomide BM $\mathrm{B}$-cell space in $\operatorname{del}(5 \mathrm{q}) \mathrm{MDS}$ was comparable to the B-cell space of age-matched healthy donors in terms of $I G H$ clonality, but showed a significantly higher somatic hypermutation rate of IGH clones, indicating an extended number of antigen-experienced B lineage cells (Figure 3A,C). $\mathrm{BM}$ (but not $\mathrm{PB}$ ) $\mathrm{B}$ lineage clonality decreased significantly even below the levels in the age-matched healthy donor group and somatic hypermutation rates of IGH clones normalized in $\mathrm{BM}$ and $\mathrm{PB}$ upon lenalidomide treatment (Figure 3A-D).

TRB clonality is increased in myelodysplastic syndrome del(5q) bone marrow and GLIPH analysis reveals the presence of myelodysplastic syndrome-specific and lenalidomide-induced T-cell clusters

The BM T-cell space of patients with del(5q) MDS showed a higher TRB clonality compared to that of healthy control BM both before and after lenalidomide treatment, while there were no differences in PB T cells suggesting antigen-driven clonal $\mathrm{T}$-cell selection only in the local BM environment (Figure 4A,B).

To further define antigen-driven T-cell dynamics in the $\mathrm{BM}$ of $\operatorname{del}(5 \mathrm{q}) \mathrm{MDS}$ patients undergoing lenalidomide treatment, we performed GLIPH analysis ${ }^{27}$ on our TRB NGS dataset. The GLIPH algorithm clusters T-cell receptors according to their presumed antigen specificity based on conserved motif analysis. ${ }^{26}$ Since our global analysis of T-cell clonality suggested antigen-driven selection in the $\mathrm{BM}$ but not in $\mathrm{PB}$, we exclusively used TRB repertoires from the local MDS microenvironment for this analysis. In a first step, T-cell clusters were determined by GLIPH analysis in BM from healthy controls as well as del(5q) MDS patients before and after lenalidomide treatment. When looking at MDS-exclusive T-cell clusters, we found a higher number of newly emerging clusters that were shared between individual patients in the samples after lenalidomide treatment compared to the clusters that were present only at the pre-treatment time point $(n=534$ vs. 240 clusters, respectively) (Figure 5A). This indicated new $\mathrm{T}$ - cell cluster generation in response to lenalidomide. Of all clusters that persisted in the BM across the two sampling time points $(n=1,242)$, the majority $(n=813)$ decreased after treatment, while only fewer clusters clonally expanded after treatment (Figure 5A,B). The few MDS-exclusive T-cell clusters that were present before exposure to lenalidomide and that expanded upon treatment were mostly clusters with high generation probability and were therefore less likely to constitute a true T-cell response directed against the MDS clone. In contrast, the T-cell clusters newly generated upon lenalidomide treatment showed much lower generation probabilities, further supporting the hypothesis that the newly emerging clusters (in contrast to the preexisting ones) are specifically generated in response to the MDS clone (Figure 5B). Together, this indicates that despite large overlaps in the pre- and post-therapeutic TRB repertoire of individual patients, lenalidomide-induced T-cell immunity may be significantly driven by newly generated T-cell clusters in the local MDS del(5q) BM microenvironment which are shared between individual MDS patients. Table 2 summarizes the presumably most specific top 25 lenalidomideinduced T-cell clusters (low generation probability) that were shared between two or more patients.

\section{Discussion}

The immunomodulatory drug lenalidomide is effective in the treatment of $\operatorname{del}(5 \mathrm{q}) \mathrm{MDS}$. While lenalidomide has undebatable direct effects on the del $(5 q)$-clone mediated by $\mathrm{CRBN}$, the immunomodulatory effects on the microenvironment are less well defined to date. In this study we used state-of-the-art NGS technology to gain insight into local BM immune environments in del(5q) MDS and their modulation upon treatment. NGS technology makes it possible to characterize large immune repertoires in depth and at high throughput, to monitor individual immune clones over time and to integrate all information to derive quantitative and reliable repertoire metrics data such as clonality indices, gene usage, clonal space distribution and somatic mutational frequencies. Thereby, the immune repertoire determined by NGS not only transforms our ability to detect imprints of antigenic drive, ${ }^{23,30}$ but also provides valuable insight into immunological drug effects. ${ }^{24,31-35}$

Our analyses showed that lenalidomide exerts systematic effects on the local B- and T-cell compartments in the $\mathrm{BM}$ of patients with $\operatorname{del}(5 q) \mathrm{MDS}$. Since no significant clonal B-cell overlap between pre- and post-treatment samples was detectable in BM, the small but highly frequent clones detected after initiation of lenalidomide treatment most likely represent newly generated clones, given that our sequencing depth of around 17,000 successfully aligned productive $I G H$ reads per sample exceeds the greatest possible number of B lineage cells in the sample (1,136-5,682 per PCR, assuming a percentage of B lineage cells with completed VDJ recombination of $3-15 \%$ of BM mononuclear cells). Collectively, the changes observed in the IGH repertoire reflect the proliferative effect on healthy B-cell precursors previously described for this 
drug. ${ }^{36}$ It is also in line with the observed upregulation of $P A X 5$, a gene involved in $V(H) D J(H)$ recombination of pro$\mathrm{B}$ cells, in lenalidomide responders described in a BM RNA sequencing study of patients with non-del(5q) MDS, suggesting that this drug non-specifically expands the $B$ lineage pool by this mechanism. ${ }^{19}$
The BM T-cell space in patients with del(5q) MDS was significantly more restricted than that of $\mathrm{PB}$ indicating $\mathrm{T}$ cell-mediated immunity in the BM niche where $T$ cells are in direct contact with the $\operatorname{del}(5 q) \mathrm{MDS}$ clone. The BM Tcell repertoire was much more conserved over time than the B-cell repertoire with a higher clonal overlap between

A

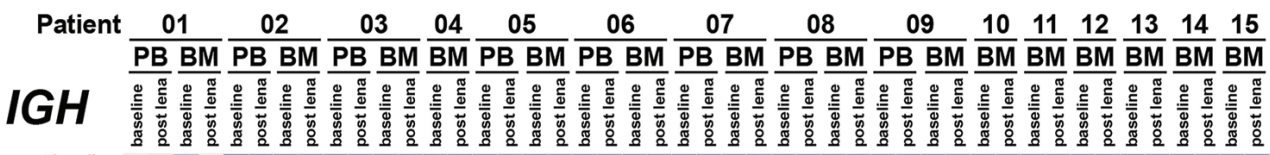

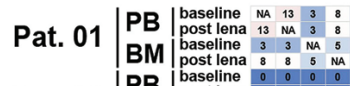

Pat. 02

Pat. 03 BM | baseline PB 占aseline

Pat. 04 BM | post lena

Pat. 05

Pat. 06

PB | $\begin{aligned} & \text { bassiline } \\ & \text { postina } \\ & \text { baseline }\end{aligned}$ BM $\mid$ bostline

6

Pat. 07 PB

Pat. 08

Pat. 09

Pat. $10 \mid \mathrm{BM}$

Pat. 11 BM baseline

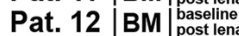

Pat. $13 \mid$ BM $\mid \begin{aligned} & \text { baseline } \\ & \text { post len }\end{aligned}$

Pat. $14 \mid$ BM | $\left.\right|_{\text {posstien }} ^{\text {baseline }}$

Pat. $15 \mid$ BM $\left.\right|_{\text {post tena }} ^{\text {base }}$

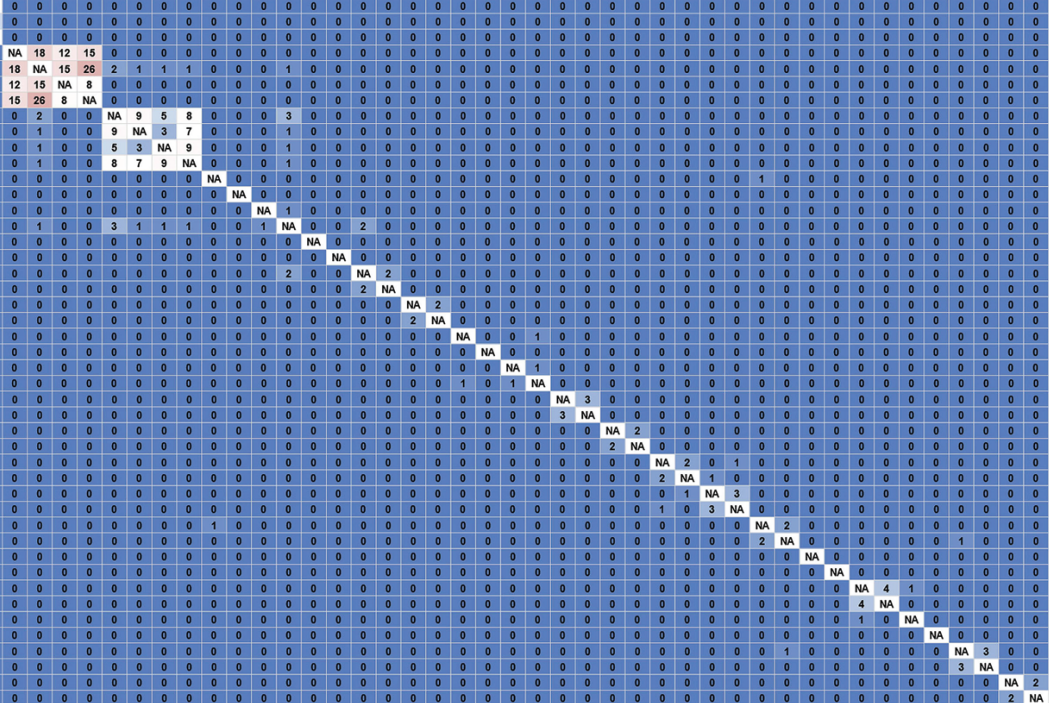

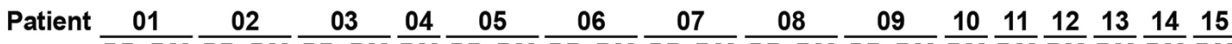

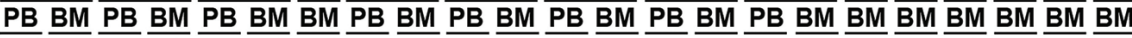
TRB

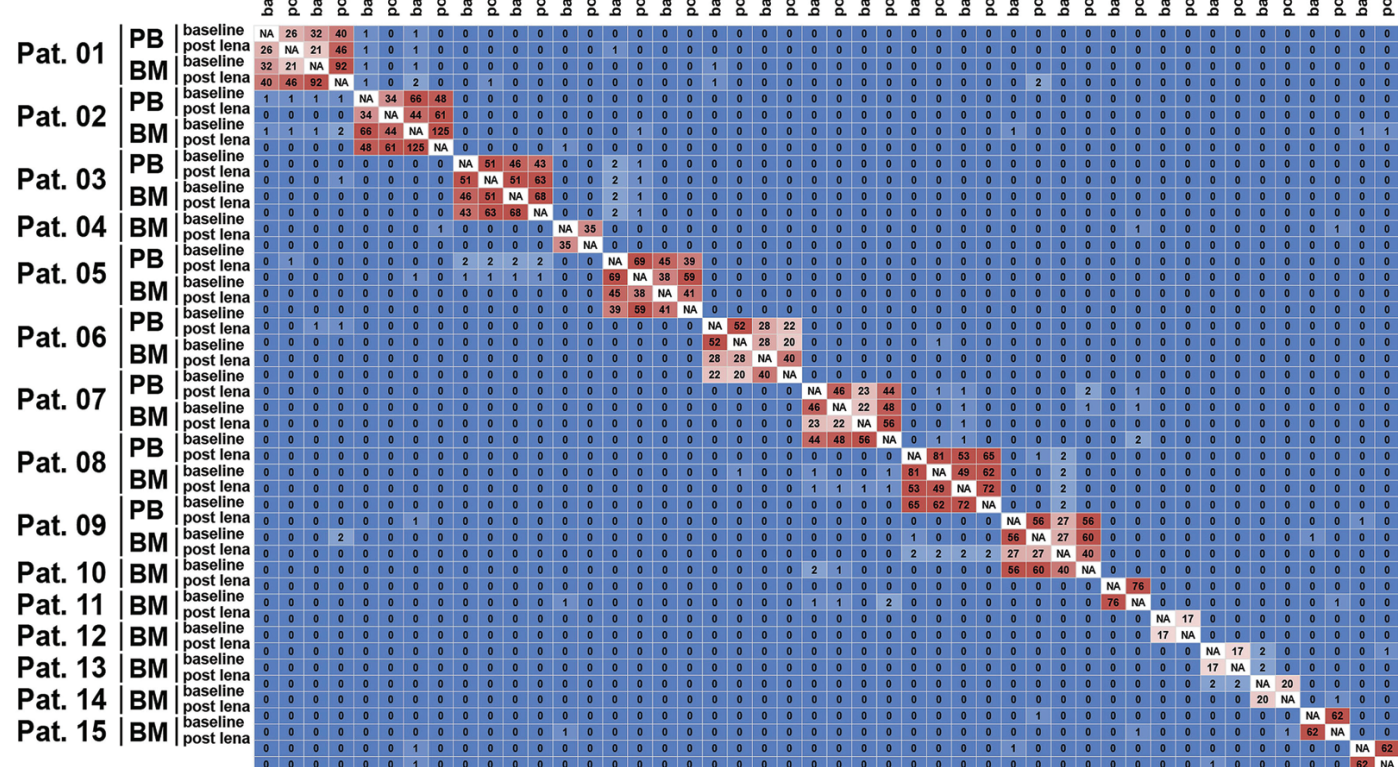

0 Shared clones

Figure 2. Clonal overlaps in T- and B-cell environments of patients with del(5q) myelodysplastic syndromes. Individual (A) IGH or (B) TRB repertoires in the peripheral blood and bone marrow of patients with del( $5 q)$ myelodysplastic syndromes before and after lenalidomide treatment were matched to each other. The absolute numbers of shared CDR3 sequences between the samples are shown. Pat.: patient; PB: peripheral blood; BM: bone marrow; MDS; myelodysplastic syndrome; baseline: prior to lenalidomide treatment; post lena: after lenalidomide treatment, NA: not analyzed. 
the pre- and post-therapeutic samples. However, when GLIPH analysis was used to determine the dynamics of Tcell clusters with presumably identical antigen recognition, we found that the majority of preexisting MDS-specific BM T-cell clusters were either lost or reduced in size, while new T-cell clusters with low to medium generation probabilities, which were shared between different patients, emerged upon lenalidomide treatment. Therefore, the cluster analysis provided indirect, but strong evidence that the observed T-cell dynamics is not
A

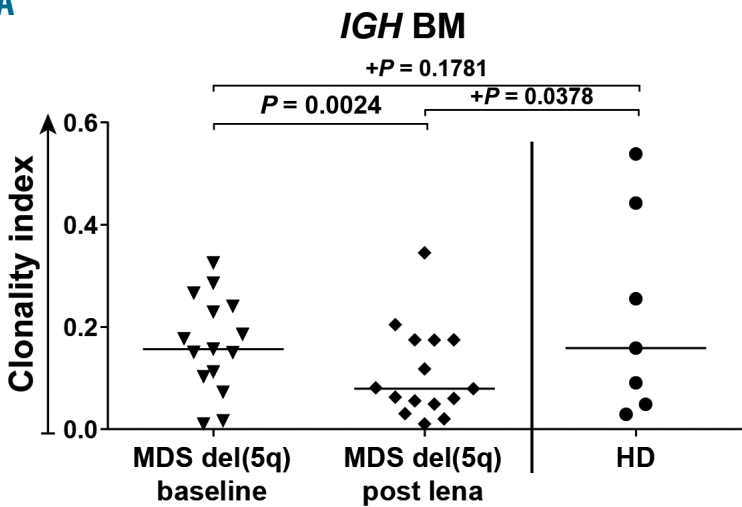

C

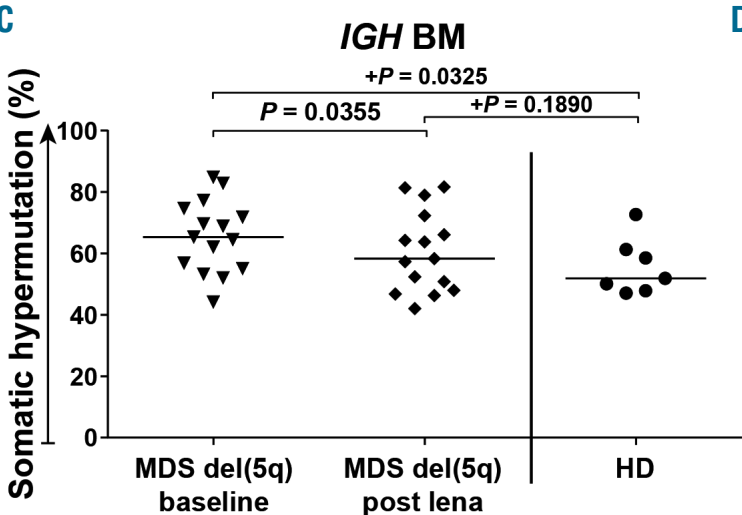

B

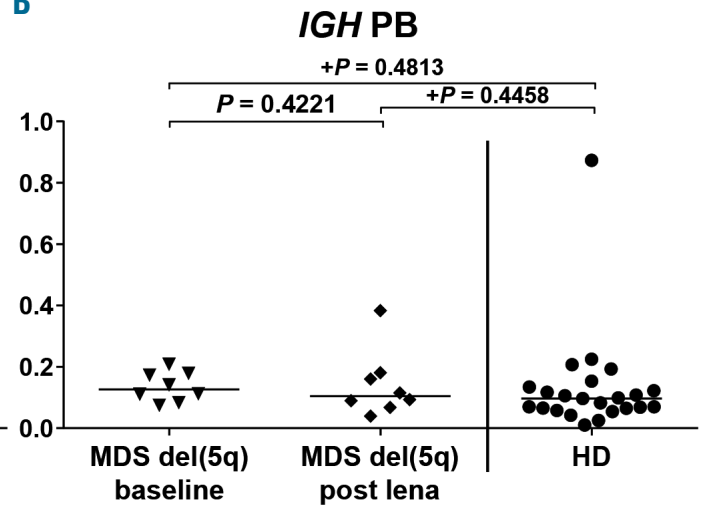

D

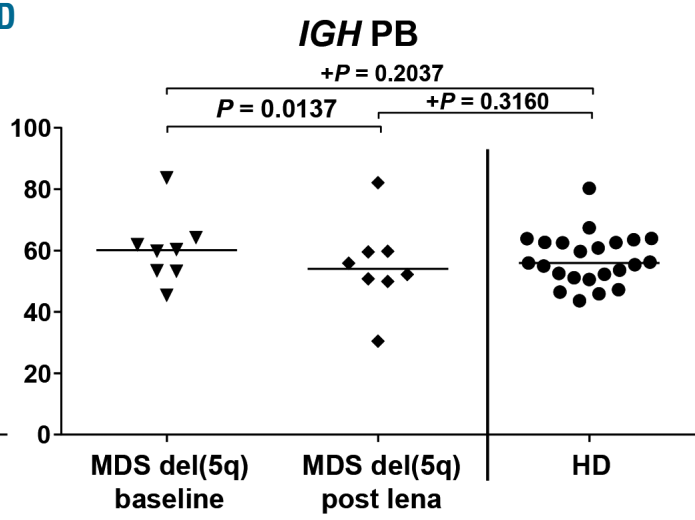

Figure 3. IGH clonality and hypermutation in the peripheral blood and bone marrow of patients with myelodysplastic syndromes at baseline and after initiation of Ienalidomide treatment. (A,B) Clonality index and (C,D) somatic hypermutation rate of the IGH repertoire in samples of (A,C) bone marrow and (B,D) peripheral blood from patients with myelodysplastic syndromes before and after lenalidomide treatment and in samples from healthy controls. The median of each group is indicated in the diagrams. Statistical analysis was performed using the Student $t$-test. Differences between pre- and post-lenalidomide samples are indicated as $P$ values and those between either pre- or post-lenalidomide samples and samples from healthy controls are indicated as $+P$ values. BM: bone marrow; PB: peripheral blood; MDS; myelodysplastic syndrome; baseline: prior to lenalidomide treatment; post lena: after lenalidomide treatment; HD: healthy donor.
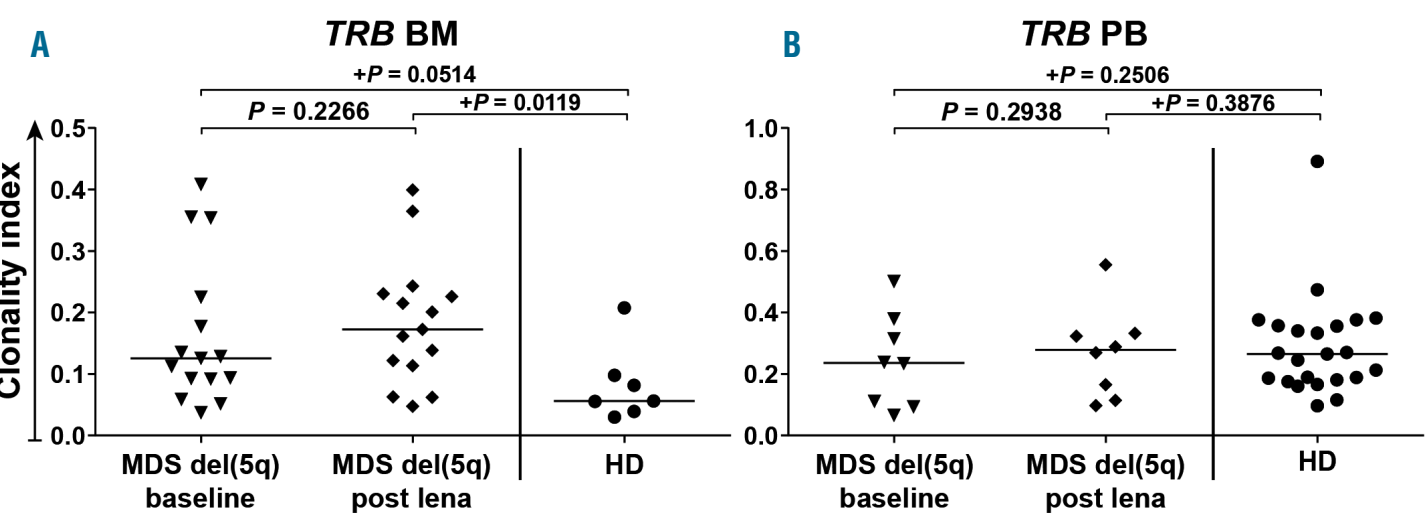

Figure 4. TRB clonality in the peripheral blood and bone marrow of patients with del $(5 q)$ myelodysplastic syndromes at baseline and after initiation of lenalidomide treatment. $(A, B)$ Clonality index of the TRB repertoire in samples of bone marrow $(A)$ and peripheral blood (B) from patients with myelodysplastic syndromes before and after lenalidomide treatment and in samples from healthy controls. The median of each group is indicated in the diagrams. Statistical analysis was performed using the Student $t$-test. Differences between pre- and post-lenalidomide samples are indicated as $P$ values and those between either pre- or post-lenalidomide samples and samples from healthy controls are indicated as $+P$ values. BM: bone marrow; PB: peripheral blood; MDS; myelodysplastic syndrome; baseline: prior to lenalidomide treatment; post lena: after lenalidomide treatment; HD: healthy donor. 
due to non-specific stimulation, but is antigen-driven and directed against the MDS del(5q) clone. Along this line of reasoning, our findings support the concept that the immunomodulatory effect of lenalidomide not only influences the functional state of $\mathrm{T}$ cells, but also induces a profound change in the local BM T-cell composition leading to the generation of novel T-cell clusters that can be observed across patients. These clusters, if confirmed by future studies, could also be helpful to explain or even predict resistance or loss of response to lenalidomide.
Moreover, it would be of interest to determine whether these clusters also appear in patients with non-del $(5 q)$ disease and represent a general mechanism of action of lenalidomide independently of the del(5q) MDS. This will need to be addressed in future studies.

Expansion of knowledge about the immune response induced by lenalidomide treatment in the BM of MDS patients may be of particular clinical importance in the era of immune checkpoint therapy. Although MDS is considered to be less immunogenic than solid tumors, the obser-

A
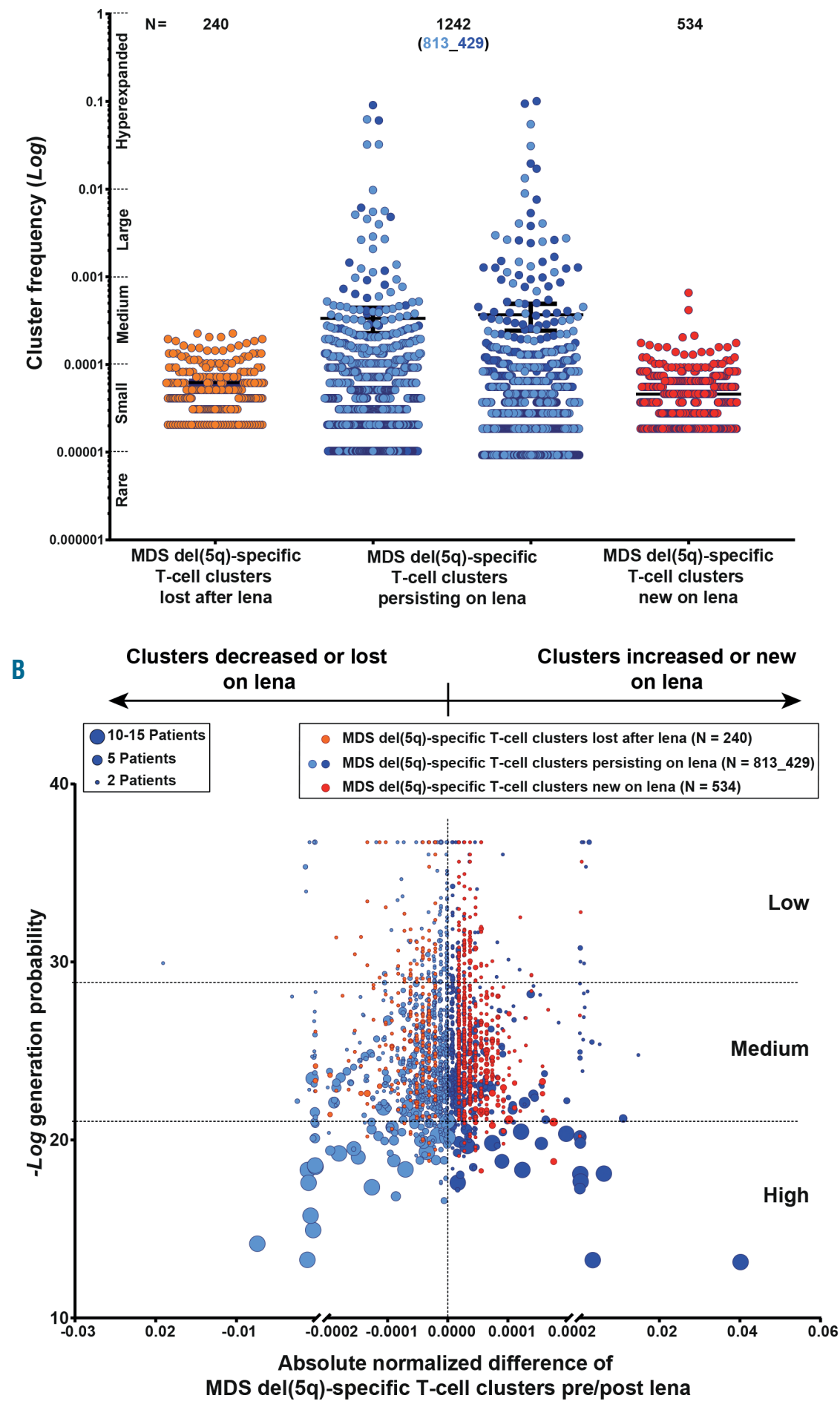

Figure 5. In silico TRB cluster analysis of del(5q) myelodysplastic syndrome bone marrow samples at baseline and after initiation of lenalidomide treatment. (A) Frequencies and (B) generation probabilities of in silico-generated TRB clusters in bone marrow mononuclear cells from patients with del(5q) myelodysplastic syndromes (MDS) at baseline versus post-lenalidomide treatment. Clusters detected in both were designated as shared and are colored according to their frequency (bright blue decreasing, dark blue increasing on treatment). The absolute normalized difference of a cluster was calculated by subtracting the cluster frequencies observed in MDS samples at baseline from frequencies observed in samples after lenalidomide treatment. The size of the dots in (B) represents the numbers of individuals sharing the specific cluster. Lena: Ienalidomide. 
Table 2. Lenalidomide-induced T-cell clusters.

\begin{tabular}{|c|c|c|c|}
\hline & Cluster (CDR3 sequence) & $\begin{array}{l}\text { apost-lenalidomide expansion } \\
\text { (absolute normalized difference) }\end{array}$ & Generation probability \\
\hline $\begin{array}{l}\text { Clusters with low generation } \\
\text { probability shared by } 3 \text { patients }\end{array}$ & $\begin{array}{c}\text { CASREPSGGKPQHF } \\
\text { CATKDRDSNQPQHF } \\
\text { CASSLWGGGDQPQHF } \\
\text { CASSLGGLAGAEQFF } \\
\text { CATSGSGRFYNEQFF }\end{array}$ & $\begin{array}{l}5.57 \mathrm{E}-05 \\
3.71 \mathrm{E}-05 \\
4.64 \mathrm{E}-05 \\
3.71 \mathrm{E}-05 \\
4.64 \mathrm{E}-05\end{array}$ & $\begin{array}{l}31.88 \\
31.23 \\
30.77 \\
30.06 \\
29.69\end{array}$ \\
\hline $\begin{array}{l}\text { Clusters with low generation } \\
\text { probability shared by } 2 \text { patients }\end{array}$ & $\begin{array}{c}\text { CASSQDFTDTQYF } \\
\text { CASTEGRADTIYF } \\
\text { CASSLGEGTAGYEQYF } \\
\text { CASSPDGGRGGEQYF } \\
\text { CAWSDRGLIGAFF } \\
\text { CASSSSQAGTEAFF } \\
\text { CASRSRDRGGDTF } \\
\text { CASSLSAGVLSEQYF } \\
\text { CASSSGPRQLFF } \\
\text { CASSLGYPGSASYKQYF } \\
\text { CASSQDSLSGYTF } \\
\text { CSVEALGGATDTQYF } \\
\text { CASSPRGVSGYTF } \\
\text { CASSFPDREQYF } \\
\text { CASSRDRGGVGEKLFF } \\
\text { CASTSGLAVTGELFF } \\
\text { CSASPRPGLAFF } \\
\text { CASRPAGGRGEQFF } \\
\text { CASTTAGAGQPQHF } \\
\text { CASSPSTGGANVLTF }\end{array}$ & $\begin{array}{l}1.86 \mathrm{E}-05 \\
1.86 \mathrm{E}-05 \\
5.57 \mathrm{E}-05 \\
5.57 \mathrm{E}-05 \\
1.86 \mathrm{E}-05 \\
2.78 \mathrm{E}-05 \\
1.86 \mathrm{E}-05 \\
3.71 \mathrm{E}-05 \\
3.71 \mathrm{E}-05 \\
6.59 \mathrm{E}-04 \\
3.71 \mathrm{E}-05 \\
1.86 \mathrm{E}-05 \\
2.78 \mathrm{E}-05 \\
1.86 \mathrm{E}-05 \\
1.86 \mathrm{E}-05 \\
2.78 \mathrm{E}-05 \\
3.71 \mathrm{E}-05 \\
4.64 \mathrm{E}-05 \\
2.78 \mathrm{E}-05 \\
4.64 \mathrm{E}-05\end{array}$ & $\begin{array}{l}36.74 \\
36.74 \\
36.74 \\
36.74 \\
36.74 \\
36.74 \\
36.74 \\
36.74 \\
36.04 \\
35.64 \\
35.64 \\
35.64 \\
34.95 \\
34.79 \\
34.43 \\
34.25 \\
34.10 \\
33.60 \\
33.56 \\
33.07\end{array}$ \\
\hline
\end{tabular}

${ }^{a}$ The absolute normalized difference is calculated by subtracting the cluster frequencies observed in baseline myelodysplastic syndrome (MDS) samples from those detected in MDS samples after lenalidomide treatment..

vation of novel lenalidomide-related T-cell clusters is reminiscent of recent data on response to PD-1 inhibitors following failure of hypomethylating agent treatment. ${ }^{37,38}$ It has been shown that hypomethylating agent therapy leads to upregulation of PD-1 and PD-L1 in MDS BM cells, which may be an explanation of the sensitivity of a subset of MDS patients to immune checkpoint therapy after failure of hypomethylating agent treatment. This concept has not yet been tested in lenalidomide-refractory MDS patients. Our data demonstrating emergence of novel Tcell clusters could, therefore, possibly serve as a novel predictive marker for such a strategy, a possibility which warrants further prospective evaluation. Identification of the antigens recognized by the described lenalidomideinduced shared T-cell clusters could help in the design of novel immunotherapeutic approaches for $\operatorname{del}(5 q)$ MDS.

\section{Acknowledgments}

The authors thank the "Hamburger Krebsgesellschaft" for financial support (grant to MB) and the Hubertus Wald Foundation for supporting a professorship $(M B)$ for immunological cancer research and treatment. The authors also thank Janina Radloff and Barbara Goesch for their excellent technical assistance and Prof. Tanja Zeller for providing relevant material. DN is an endowed Professor of the Deutsche Jose Carreras Leukemia Stiftung (DJCLSHO3/01).

\section{References}

1. Haase D, Germing U, Schanz J, et al. New insights into the prognostic impact of the karyotype in MDS and correlation with subtypes: evidence from a core dataset of 2124 patients. Blood. 2007;110(13):4385-4395.

2. Kuendgen A, Lauseker M, List AF, et al. Lenalidomide does not increase AML progression risk in RBC transfusion-dependent patients with Low- or Intermediate-1-risk MDS with $\operatorname{del}(5 q)$ : a comparative analysis. Leukemia. 2013;27(5):1072-1079

3. Boultwood J, Pellagatti A, Cattan $\mathrm{H}$, et al. Gene expression profiling of CD34+ cells in patients with the $5 \mathrm{q}-$ syndrome. $\mathrm{Br} \mathrm{J}$ Haematol. 2007;139(4):578-589.

4. Ebert BL, Pretz J, Bosco J, et al. Identification of RPS14 as a 5q- syndrome gene by RNA interference screen. Nature. 2008;451(7176): 335-339.

5. Boultwood J, Pellagatti A, McKenzie AN Wainscoat JS. Advances in the 5q- syndrome. Blood. 2010;116(26):5803-5811.

6. Dutt S, Narla A, Lin K, et al. Haploinsufficiency for ribosomal protein genes causes selective activation of p53 in human erythroid progenitor cells. Blood. 2011;117(9):2567-2576.

7. Wei S, Chen X, Rocha K, et al. A critical role for phosphatase haplodeficiency in the selective suppression of deletion $5 q$ MDS by enalidomide. Proc Natl Acad Sci U S A. 2009;106(31):12974-12979.

8. Pellagatti A, Jadersten M, Forsblom AM, et al. Lenalidomide inhibits the malignant clone and up-regulates the SPARC gene mapping to the commonly deleted region in $5 q$ - syndrome patients. Proc Natl Acad Sci U
S A. 2007;104(27):11406-11411

9. Heise C, Carter T, Schafer P, Chopra R Pleiotropic mechanisms of action of lenalidomide efficacy in $\operatorname{del}(5 q)$ myelodysplastic syndromes. Expert Rev Anticancer Ther. 2010;10(10):1663-1672.

10. Fenaux P, Giagounidis A, Selleslag D, et al. A randomized phase 3 study of lenalidomide versus placebo in RBC transfusion-dependent patients with Low-/Intermediate-1-risk myelodysplastic syndromes with del5q Blood. 2011;118(14):3765-3776.

11. Giagounidis A, Mufti GJ, Mittelman M, et al. Outcomes in RBC transfusion-dependent patients with Low-/Intermediate-1-risk myelodysplastic syndromes with isolated deletion $5 \mathrm{q}$ treated with lenalidomide: a subset analysis from the MDS-004 study. Eur J Haematol. 2014:93(5):429-438.

12. Kronke J, Fink EC, Hollenbach PW, et al. 
Lenalidomide induces ubiquitination and degradation of CK1alpha in del(5q) MDS. Nature. 2015;523(7559):183-188.

13. Kronke J, Udeshi ND, Narla A, et al. Lenalidomide causes selective degradation of IKZF1 and IKZF3 in multiple myeloma cells. Science. 2014;343(6168):301-305.

14. Eichner R, Heider M, Fernandez-Saiz V, et al. Immunomodulatory drugs disrupt the cereblon-CD147-MCT1 axis to exert antitumor activity and teratogenicity. Nat Med. 2016;22(7):735-743.

15. Staffler G, Szekeres A, Schutz GJ, et al. Selective inhibition of $\mathrm{T}$ cell activation via CD147 through novel modulation of lipid rafts. J Immunol. 2003;171(4):1707-1714.

16. Hu J, Dang N, Yao H, et al. Involvement of HAb18G/CD147 in T cell activation and immunological synapse formation. J Cell Mol Med. 2010;14(8):2132-2143.

17. Landskron J, Tasken K. CD147 in regulatory T cells. Cell Immunol. 2013;282(1):17-20.

18. McDaniel JM, Zou JX, Fulp W, Chen DT, List AF, Epling-Burnette PK. Reversal of Tcell tolerance in myelodysplastic syndrome through lenalidomide immune modulation. Leukemia. 2012;26(6):1425-1429.

19. Kerdivel G, Chesnais V, Becht E, et al. Lenalidomide-mediated erythroid improvement in non-del $(5 q)$ myelodysplastic syndromes is associated with bone marrow immuno-remodeling. Leukemia. 2018;32(2): 558-562.

20. Schuler E, Giagounidis A, Haase D, et al. Results of a multicenter prospective phase II trial investigating the safety and efficacy of lenalidomide in patients with myelodysplastic syndromes with isolated del(5q) (LEMON 5). Leukemia. 2016;30(7):1580-1582.

21. Mossner M, Jann IC, Nowak D, et al. Prevalence, clonal dynamics and clinical impact of TP53 mutations in patients with myelodysplastic syndrome with isolated deletion $(5 q)$ treated with lenalidomide: results from a prospective multicenter study of the German MDS Study Group (GMDS). Leukemia. 2016;30(9):1956-1959.

22. van Dongen JJ, Langerak AW, Bruggemann $\mathrm{M}$, et al. Design and standardization of PCR primers and protocols for detection of clonal immunoglobulin and T-cell receptor gene recombinations in suspect lymphoproliferations: report of the BIOMED-2 Concerted Action BMH4-CT98-3936. Leukemia. 2003;17(12):2257-2317

23. Schliffke S, Akyuz N, Ford CT, et al. Clinical response to ibrutinib is accompanied by normalization of the T-cell environment in CLLrelated autoimmune cytopenia. Leukemia. 2016;30(11):2232-2234

24. Akyuz N, Brandt A, Stein A, et al. T-cell diversification reflects antigen selection in the blood of patients on immune checkpoint inhibition and may be exploited as liquid biopsy biomarker. Int J Cancer. 2017;140 (11):2535-2544

25. Bolotin DA, Poslavsky S, Mitrophanov I, et al. MiXCR: software for comprehensive adaptive immunity profiling. Nat Methods. 2015;12(5):380-381

26. Glanville J, Huang $\mathrm{H}, \mathrm{Nau} A$, et al. Identifying specificity groups in the $T$ cell receptor repertoire. Nature. 2017;547(7661): 94-98.

27. Marcou O, Mora T, Walczak AM. Highthroughput immune repertoire analysis with IGoR. Nat Commun. 2018;9(1):561.

28. Kirsch I, Vignali M, Robins H. T-cell receptor profiling in cancer. Mol Oncol. 2015;9(10):2063-2070.

29. Pielou EC. Species-diversity and patterndiversity in the study of ecological succession. J Theor Biol. 1966;10(2):370-383.
30. Schliffke S, Sivina M, Kim E, et al. Dynamic changes of the normal $B$ lymphocyte repertoire in CLL in response to ibrutinib or FCR chemo-immunotherapy. Oncolmmunology. 2017;e1417720

31. Tumeh PC, Harview CL, Yearley JH, et al PD-1 blockade induces responses by inhibiting adaptive immune resistance. Nature. 2014;515(7528):568-571.

32. Riaz N, Havel JJ, Makarov V, et al. Tumor and microenvironment evolution during immunotherapy with nivolumab. Cell. 2017;171(4):934-949.

33. Gros A, Parkhurst MR, Tran E, et al Prospective identification of neoantigen-specific lymphocytes in the peripheral blood of melanoma patients. Nat Med. 2016;22(4): 433-438.

34. Robert L, Tsoi J, Wang X, et al. CTLA4 blockade broadens the peripheral T-cell receptor repertoire. Clin Cancer Res. 2014;20(9):2424-2432.

35. Cha E, Klinger M, Hou Y, et al. Improved survival with $\mathrm{T}$ cell clonotype stability after anti-CTLA-4 treatment in cancer patients. Sci Transl Med. 2014;6(238):238ra270.

36. Verhelle D, Corral LG, Wong K, et al Lenalidomide and CC-4047 inhibit the proliferation of malignant $\mathrm{B}$ cells while expanding normal CD34+ progenitor cells. Cancer Res. 2007;67(2):746-755

37. Boddu P, Kantarjian H, Garcia-Manero G Allison J, Sharma P, Daver N. The emerging role of immune checkpoint based approaches in AML and MDS. Leuk Lymphoma. 2018;59(4):790-802.

38. Hidalgo-Lopez JE, Kanagal-Shamanna R, Quesada AE, et al. Progress in myelodysplastic syndromes: clinicopathologic correlations and immune checkpoints. Clin Lymphoma Myeloma Leuk. 2017;17s:S16-S25. 